\title{
An amino acid to SORT the brain's proteome
}

Nat. Biotechnol. 36, 156-159 (2018)

When it comes to understanding a cell's function and characteristics, one of the keys is its proteome-that is, the identity of all of the proteins that the cell manufactures. Any individual cell type only creates a subset of all of the proteins encoded by its DNA, and this proteomic fingerprint is part of what makes cell types unique.

But researchers have struggled to measure proteomes accurately. There are methods to tag proteins and identify them, but current approaches that rely on removing a cell from its surroundings or growing it in culture can alter the nature of the proteome itself. Cells may be physically damaged by shear forces as a flow cytometer deflects a cell of interest out of a flowing stream of cells. A cell grown in a petri dish may respond to its new environment by altering the proteins it produces, or it may evolve over generations. In any case, the cell has been ripped away from the neighboring cells and in vivo environment that may also shape its identity and proteome.

To best understand a cell's proteome, researchers would like to be able to analyze it directly, whether in a tissue slice, or in a whole animal. In a study published in Nature Biotechnology, researchers unveiled a technique to do just that, using a novel amino acid.

The technique arose over coffee between colleagues at the Medical Research Council Laboratory of Molecular Biology in Cambridge, England. The spot is a frequent incubator for cross-disciplinary research, says Russ Ernst, who was a post-doc at the time, working in a biochemistry lab that was developing novel amino acids. He discussed his work with Toke Krogager, a post-doc working in circadian biology who was trying to get at proteomes in mouse brains. "We wanted to do something interesting with this (novel amino acid) technology, and it happened to fit with a need they had already identified," says Ernst, who is now a research scientist at Kymab (Cambridge, UK).

The technique relies on an adenoassociated virus to deliver to the target cell the machinery responsible for incorporating a new amino acid into growing proteins. Existing novel amino acids were too expensive to be practical, so Ernst and his colleagues developed a new, cheaper, one, called AlkK, which would be suitable for use in mice. The new amino acid uses the same genetic code as the natural amino

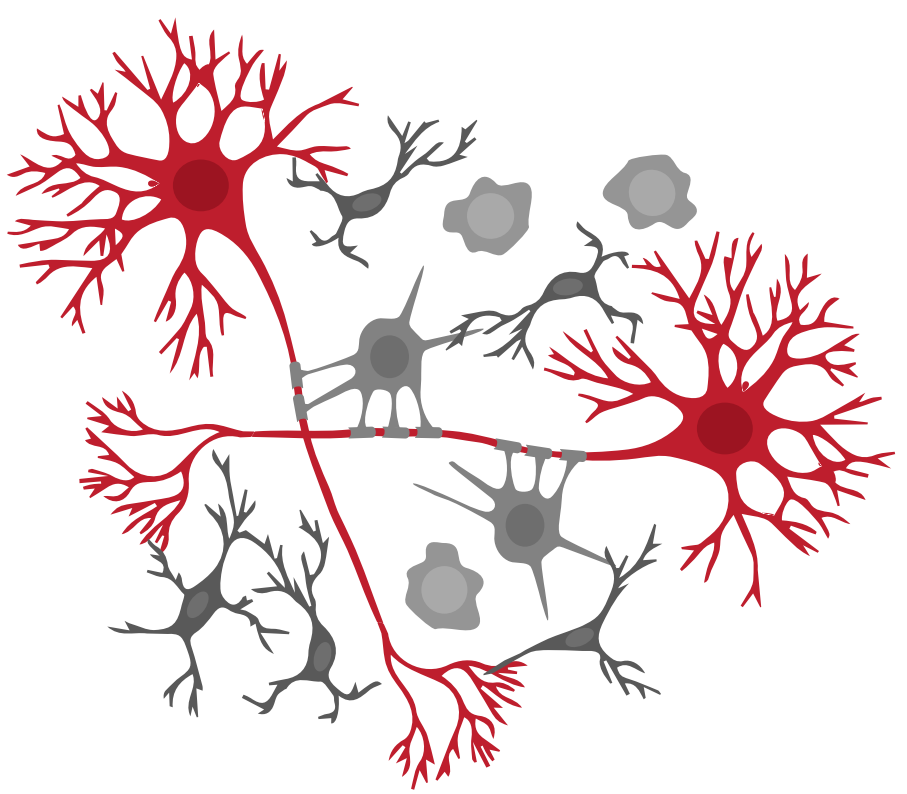

Labeling proteins associated with a targeted cell type in the brain. Credit: Image adapted from Nat. Biotechnol. 36, 156-159 (2018)

acid methionine. Most of the time, the cell's native machinery inserts methionine, but occasionally the AlkK-bearing transfer RNA slips in instead and incorporates AlkK into the growing protein instead of methionine.

A chemical tag that reacts with AlkK can then be used to isolate and identify the species of protein made by the cell. In the absence of AlkK, the transfer RNA and other machinery lay idle, but AlkK can be delivered through the animal's drinking water, giving researchers control over the timing of production of the labeled proteins.

It also allows researchers to query a specific type of cell in a defined region of the brain, because they can expose only those cells to the virus and the novel amino acid machinery it carries. This contrasts with other recently described methods, in which the animals are genetically engineered to express the tagging machinery more broadly (Nat Biotechnol. 35, 1196-1201; 2017). "This gives you independent spatial and genetic control, versus having a transgenic mouse where the (machinery) is expressed in every cell of a particular subtype," says Ernst.

An important caveat is that insertion of an unnatural amino acid, or the presence of the insertion machinery, could affect the proteome or the function of the cell. If it does, then the measurement still doesn't capture the fingerprint of the cell in its natural state.
Ernst was surprised to learn that his neurobiology colleagues weren't worried about it. That's because in their experiments, the neurobiologists found that mice containing the unnatural amino acid machinery in the suprachiasmatic nucleus (SCN) could still maintain their internal clocks. That's notable because the SCN is responsible for its circadian rhythms. The occasional substitution of AlkK for methionine couldn't be drastically affecting cell function. "That was basically case closed to our neurobiology collaborators, so that's what convinced us that the system didn't disrupt physiological function," says Ernst.

He now hopes that the method will help researchers get a better handle on the molecular underpinnings of learning and memory. This could be particularly useful in mice, where many behavioral assays are already established. "The dream would be to use this work to start to bridge the gap between behavioral neuroscience and molecular neuroscience. That's an overstatement, but the hope is that we could start to eat away at the edge of that gap," says Ernst.

Jim Kling

Published online: 26 March 2018 https://doi.org/10.1038/s41684-018-0041-8 